\title{
Special Issue: highlights from the IEEE Latin American Symposium on Circuits and Systems (LASCAS) 2015
}

\author{
José M. de la $\operatorname{Rosa}^{1}$ - Carlos Galup-Montoro ${ }^{2}$ - Fernando Silveira ${ }^{3}$. \\ Alfredo Arnaud ${ }^{4}$
}

Published online: 29 September 2016

(c) Springer Science+Business Media New York 2016

We are very pleased to present to the readers of the Springer Journal on Analog Integrated Circuits and Signal Processing (ALOG) a selection of extended papers from the 6th edition of the IEEE Latin America Symposium on Circuits and Systems (LASCAS 2015). The symposium, a forum for discussion of the latest technical novelties on circuits and systems topics, took place in MontevideoUruguay, organized by the local IEEE-CAS chapter. LASCAS is an annual meeting that brings together researchers, industry, engineers, students interested in circuits and systems across a wide spectrum of scientific and technological fields: VLSI, analog and digital signal processing, biomedical circuits and systems, multimedia systems, nanoelectronics, neural networks, communications

José M. de la Rosa

jrosa@imse-cnm.csic.es

Carlos Galup-Montoro

carlos@eel.ufsc.br

Fernando Silveira

silveira@fing.edu.uy

Alfredo Arnaud

aarnaud@ucu.edu.uy

1 Institute of Microelectronics of Seville, IMSE-CNM (CSIC/ University of Seville), C/Américo Vespucio S/N, CP 41092 Seville, Spain

2 Department of Electrical and Electronics Engineering, Federal University of Santa Catarina, Campus UniversitarioTrindade, Florianópolis, SC 88040-900, Brazil

3 Instituto de Ingeniería Eléctrica, School of Engineering, Universidad de la República, Julio Herrera y Reissig 565, CP 11300 Montevideo, Uruguay

4 Electrical Engineering Department, Universidad Católica del Uruguay, Av. 8 de Octubre 2801, CP 11600 Montevideo, Uruguay circuits, CAD, power electronic circuits, sensors, among others. The conference is a great opportunity for colleagues operating in very different areas of industrial, scientific, and technological endeavor to come together and create the basis for renewed collaboration.

In 2015, more than 180 people attended LASCAS including university students and academics, researchers, and engineers from the industry, from 26 countries and 3 continents. 160 papers were submitted to the conference. After a thorough review process 93 papers were accepted for presentation at the conference and publication in the IEEE LASCAS Proceedings, making up a high-quality technical program covering a wide variety of subjects in the field of circuits and systems. Based on the evaluations of the Program Committee members and the corresponding session chairs, a total of 20 of these works were invited to submit a substantially extended and revised manuscript to this Special Issue of LASCAS 2015. These papers went through a second-round of peer-review process and 12 papers were finally selected for this Special Issue.

In the analog circuit design domain, the papers deal with voltage references: A Computer-Aided Approach for Voltage Reference Circuit Design, by Fabian Olivera et al., micropower circuits: Low-Power Operational Transconductance Amplifier with Slew-Rate Enhancement Based on Non-Linear Current Mirror, by Pablo Pérez Nicoli et al., and Step Down DC/DC converter for Micro-Power Medical Applications, by Matias Miguez et al., biomedical circuits: High Stability Voltage Controlled Current Source for Cervical Cancer Detection using Electrical Impedance Spectroscopy, by José A. Amaya Palacio et al., or sensor circuits: Front-end for gaseous detectors read-out with improved PSRR in $130 \mathrm{~nm}$ CMOS technology by Hugo Hernández et al. Three papers address topics on RF and communication circuits: A charge transfer-based high 
performance, ultra-low power CMOS charge pump for PLLs, by Susan M. Schober et al., Design Optimization of a CMOS RF Detector, by Nicolás Barabino et al., and $A$ CMOS Low Noise Transconductance Amplifier for 1-6 GHz Bands, by David Cordova et al. One paper analyzes techniques for AD converters: Design methodology for low-jitter differential clock recovery circuits in high performance ADCs, by Juan Nuñez et al., while device modeling with analog application is considered in $A$ Compact Model for Flicker Noise in MOSFETs considering both correlated mobility and carrier number fluctuations, by Alfredo Arnaud et al. Neural Networks are examined in Implementing Delay Insensitive Oscillatory Neural Networks using CMOS and Emerging Technology, by Thomas Christopher Jackson, and network-on-chip (NoC) circuits in A New Local Clock Generator for Globally Asynchronous Locally Synchronous MPSoCs by Guilherme Heck et al.

We deeply thank the authors that prepared the extended papers for this Special Issue, and the reviewers for their contribution to the creation of the LASCAS 2015 technical program and for their involvement in the revision of the selected papers for this Special Issue. We thank the session chairs who not only contributed to the successful development of the symposium, but also helped in the selection of the best papers for journal publication. Finally we would like to express our appreciation to Dr. Mohammed Ismail, Editor-in-chief of the Journal, for the opportunity to organize this Special Issue, and to the assistance of Raja Chinnadurai and Ayshwarya Ganesan, from the Springer Journal Editorial Office, for their diligent work and help. We hope you appreciate this Special Issue.

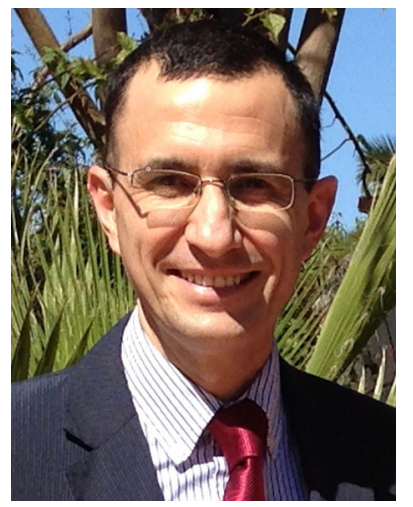

José M. de la Rosa received the M.S. degree in Physics in 1993 and the Ph.D. degree in Microelectronics in 2000, both from the University of Seville, Spain. Since 1993 he has been working at the Institute of Microelectronics of Seville (IMSE), which is in turn part of the Spanish Microelectronics Center (CNM) of the Spanish National Research Council (CSIC), where he heads a research group on micro/nanoelectronic circuits and systems. $\mathrm{He}$ is also with the Department of Electronics and Electromagnetism of the University of Seville, where he is currently a Professor. His main research interests are in the field of analog and mixed-signal integrated circuits, including analysis, behavioral modeling, and design automation of such circuits. In these topics, Dr. de la Rosa has participated in a number of National and European research and industrial projects, and has co-authored more than 200 international peer-reviewed publications, including journal and conference papers, book chapters and the books Systematic Design of CMOS Switched-Current Bandpass Sigma-Delta Modulators for Digital Communication Chips (Kluwer 2002), CMOS Cascade Sigma-Delta Modulators for Sensors and Telecom: Error Analysis and Practical Design (Springer 2006), Nanometer CMOS Sigma-Delta Modulators for Software Defined Radio (Springer, 2011) and CMOS Sigma-Delta Converters: Practical Design Guide (Wiley-IEEE Press 2013). Dr. de la Rosa is an IEEE Senior Member, Member of the Executive Committee of the IEEE-Spain Section, and a member of the Analog Signal Processing Technical Committee of the IEEE Circuits and Systems Society, where he serves as the Chair of the Spanish Chapter. He served as Associate Editor for IEEE Transactions on Circuits and Systems I: Regular Papers (2012-2015), where he received the 2012-2013 Best Associate Editor Award and served as Guest Editor of the Special Issue on Custom Integrated Circuits Conference (CICC) in 2013 and 2014. He served also as Guest Editor of the Special Issue of the IEEE Journal on Emerging and Selected Topics in Circuits and Systems on Next-Generation Delta-Sigma Converters. He is also a member of the Steering Committee of IEEE MWSCAS. He participated and is currently participating in the organizing, steering and technical committees of diverse international conferences, among others IEEE ISCAS, MWSCAS, IEEE ICECS, IEEE LASCAS, IFIP/IEEE VLSI-SoC and DATE, and served as TPC chair of IEEE MWSCAS 2012, IEEE ICECS 2012 and IEEE LASCAS 2015. He is currently serving as Deputy Editor in Chief of the IEEE Transactions on Circuits and Systems-II: Express Briefs for the term 2016-2017.

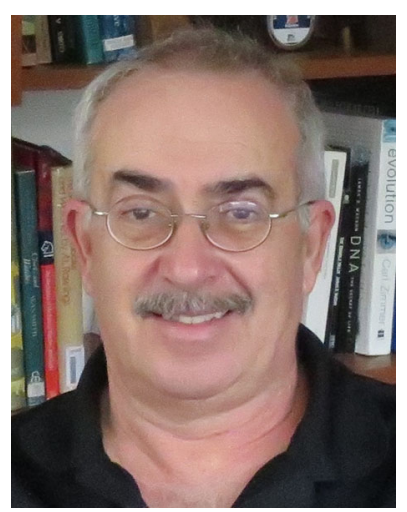

Carlos Galup-Montoro studied engineering sciences at Universidad de la República-Uruguay, and electronic engineering at the National Polytechnic School of Grenoble (INPG), France. He received an engineering degree in electronics in 1979 and a doctorate degree in 1982, both from INPG. From 1982 to 1989 he was with the University of São Paulo, Brazil, where he was engaged in junction field effect transistor (JFET) fabrication and analog circuit design. Since 1990, he has been with the Electrical Engineering Department, Federal University of Santa Catarina, Florianópolis, Brazil where he is now a professor. In the second semester of the academic year 1997/98 he was a research associate with the Analog Mixed Signal Group, Texas A\&M University. In the academic year 2008/09 2009 he was a visiting scholar at UC Berkeley. He is coauthor of the textbooks: "MOSFET Modeling for Circuit Analysis and Design", World Scientific, 2007 and "CMOS Analog Design Using All-Region MOSFET Modeling", Cambridge University Press, 2010. His main research interests and expertise are in field effect transistor and sensor modeling and analog and mixed signal circuit design. 


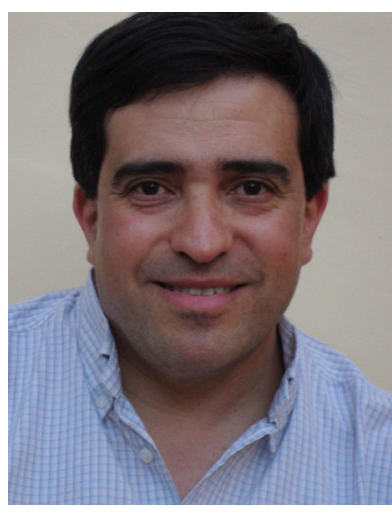

Fernando Silveira Fernando Silveira received the Electrical Engineering degree from Universidad de la República, Uruguay in 1990 and the M.Sc. and Ph.D. degree in Microelectronics from Universite catholique de Louvain, Belgium in, respectively, 1995 and 2002. He is Professor at Universidad de la Republica, Uruguay, where he is currently the Head of the Electrical Engineering Department. His research interests are in design of ultra low-power analog and RF integrated circuits and systems, in particular with biomedical application. In this field, he is co-author of one book and many technical articles and advised Masters and Ph.D. thesis. He has acted as invited plenary speaker at various events and served as reviewer and member of the TPC of several journals and conferences. He has multiple industrial activities with CCC Medical Devices and NanoWattICs, including leading the design of an ASIC for implantable pacemakers, applied in industrial production and designing analog circuit modules for implantable devices for various companies worldwide (USA, Israel, Europe and Canada). These devices, which are currently on the market or under human clinical evaluation, are mainly related to the cardiovascular and neural fields. Dr. Silveira received the Distinguished Engineer award by the Uruguayan Association of Engineers in 2007 and was a member for 2011-2012 of the Distinguished Lecturers Program of the IEEE Circuits and Systems Society.

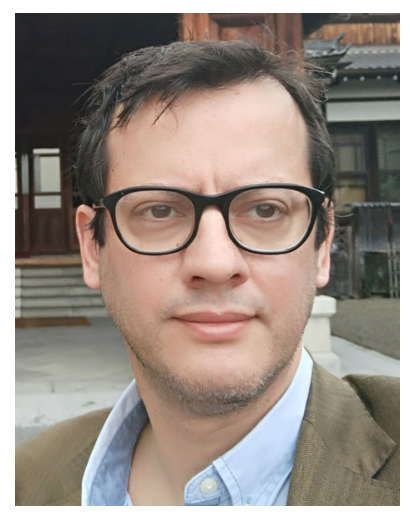

Alfredo Arnaud Alfredo Arnaud received the Ph.D. and M.Sc. in microelectronics from Universidad de la RepúblicaUruguay, in 2000 and 2004 respectively. Since 2004 he joined Electrical Engineering Department at Universidad Católica del Uruguay, where he started the $\mu \mathrm{DIE}$ research group. His area of expertise is analog and mixedmode circuit design (low-noise, low-voltage, low power design) for implantable medical, and portable applications including RFID, medical, and compact/portable POS devices, and MOS transistor modeling (noise, mismatch). Dr. Arnaud published more than 70 papers in international journals and scientific meetings and participated in more 20 funded R\&D projects. He is the co-founder of two technology companies in Latin America: BQN in 2004 dedicated to electronics and software development for POS, medical, RFID devices, and traceability within de agribusiness industry, and Chipmate in 2009 a spin-off of the $\mu \mathrm{DIE}$ aimed at the design of ASICs for medical devices and others. For the industry, he led the design teams of three ASICs for implantable medical devices, and embedded electronics like portable POS equipment, a USB dynamic medical scale, and a portable RFID reader according to ISO11784/11785 standard. 